\title{
Retinal Pigment Epithelium Tear after Vitrectomy for Vitreomacular Traction Syndrome in an Eye with Retinal Angiomatous Proliferation
}

\author{
Takayuki Baba Juntaro Uehara Masayasu Kitahashi \\ Hirotaka Yokouchi Mariko Kubota-Taniai Toshiyuki Oshitari \\ Shuichi Yamamoto
}

Department of Ophthalmology and Visual Science, Chiba University Graduate School of Medicine, Chiba, Japan

\section{Key Words}

Retinal angiomatous proliferation · Vitreomacular traction syndrome · Vitrectomy · Retinal pigment epithelium tear

\begin{abstract}
An 87-year-old Japanese man presented with retinal angiomatous proliferation (RAP) and a retinal pigment epithelium (RPE) detachment in his right eye. His decimal best-corrected visual acuity was 0.15 in the right eye, and optical coherence tomography (OCT) showed a vitreomacular adhesion in the right eye as well. After 3 monthly intravitreal injections of ranibizumab, the size and height of the RPE detachment was significantly reduced. The accumulated intra- and subretinal fluid also disappeared, but the vitreomacular traction remained. Pars plana vitrectomy was performed, and the posterior hyaloid was separated from the retina with a vitrectomy cutter without any intraoperative complications. Two months after the surgery, a large RPE tear was observed over the macular area. His visual acuity decreased to 0.06 and remained unchanged thereafter. We suggest that the small tear led to the larger RPE tear because vitreomacular traction was transmitted to the RPE through the fibrovascular tissue of the RAP during the creation of the hyaloid detachment. Because such an RPE tear has not been reported after vitrectomy for vitreomacular traction, surgeons need to pay special attention to this potential complication in eyes with vitreomacular traction and RAP.


Baba et al.: Retinal Pigment Epithelium Tear after Vitrectomy for Vitreomacular Traction Syndrome in an Eye with Retinal Angiomatous Proliferation

\section{Introduction}

Retinal angiomatous proliferation (RAP) [1] is a disease associated with age-related macular degeneration (AMD) and is a major cause of blindness in the elderly. The mechanism that causes the development of neovascular lesions in eyes with wet-type AMD has been extensively investigated. There are many factors associated with the development of AMD, and an adhesion of the posterior vitreous cortex to the retina, vitreomacular traction, has been reported to be one of the causes [2]. An incomplete posterior vitreous separation has been reported to be associated with a progression of choroidal neovascularization (CNV), but this association has not been observed in eyes with polypoidal choroidal vasculopathy [3].

Anteroposterior traction on the macula by the vitreous cortex worsens neovascular lesions in AMD cases by shearing forces on the retinal cells and retinal pigment epithelium (RPE), activating Muller cells, causing ischemia due to reduced oxygenation of the macular area, and inflammation [4]. However, there is no information on the relationship between the attachment of the posterior hyaloid and the progression of RAP.

Currently, the standard treatment for wet AMD is an intraocular injection of antivascular endothelial growth factor (VEGF). Anti-VEGF drugs can block leakage from neovascular lesions and reduce the size of the CNV in eyes with wet AMD. The efficacy of anti-VEGF therapy has been reported to be less in wet AMD cases without posterior vitreous separation [5]. This has been attributed to a chronic tractional force caused by the posterior hyaloid that keeps the CNV active and results in a resistance to the anti-VEGF therapy.

A firm attachment of the posterior hyaloid and macula has been reported during vitreous surgery [6-8]. In most cases, detaching the posterior hyaloid leads to a regression of the $\mathrm{CNV}$ and improvement of vision even though the neovascular membrane is not excised. Macular complications were not observed in these reports, and the surgical technique appeared to be relatively safe.

Here, we report our findings in a case of RAP that developed a tear of the RPE after vitrectomy was performed in order to release a vitreomacular adhesion.

\section{Case Report}

An 87-year-old Japanese man complained of a gradually progressive vision decrease in his right eye. He reported that he was being treated for mild hypertension. His ocular history revealed that he had untreated neovascular AMD in his left eye and open-angle glaucoma in both eyes. His best-corrected visual acuity was 0.15 in both eyes, and the intraocular pressure was $14 \mathrm{~mm} \mathrm{Hg}$ in both eyes with latanoprost eye drops. Slit-lamp examination showed no abnormalities except for moderate cataracts in both eyes.

Ophthalmoscopy showed a shallow RPE detachment and macular edema accompanied by multiple drusen in his right eye (fig. 1a). His left eye had advanced AMD with a subretinal scar and severe macular edema. Optical coherence tomography (OCT) showed an RPE detachment, intraretinal hyperreflectivity and edema (fig. 1b). Vitreoretinal traction was also evident (fig. 1c). Fluorescein angiography showed a pooling of dye corresponding to the RPE detachment (fig. 1d). A hyperfluorescent spot suggesting a retinal choroidal anastomosis was observed by indocyanine green angiography (fig. 1e). He was diagnosed with RAP in the right eye. 
After 3 monthly injections of intravitreal ranibizumab, the RPE detachment was resolved (fig. 2), and his visual acuity slightly improved to 0.2 . Macular traction still remained, and we believed that vitreomacular traction limited the visual recovery.

After discussing the rationale for vitreous surgery to remove vitreous traction and possible complications, the patient agreed to undergo surgery. Pars plana vitrectomy was performed using a 23-gauge system with cataract extraction and implantation of an intraocular lens. The posterior vitreous was separated from the retina using triamcinolone acetonide to make the vitreous more visible. We noted that the posterior hyaloid was firmly attached to the macular area of the retina, and it was carefully separated by aspiration with the vitreous cutter. The internal limiting membrane was not removed during surgery. No intraoperative complications were noted.

One month after the surgery, his visual acuity was 0.1 with normal foveal depression and no intraretinal fluid. Two months after the surgery, a large tear of the RPE was found over the entire macula (fig. 3). The detached RPE contracted toward the angiomatous lesion. Although the patient did not notice any decrease in his vision, there was significant recurrence of the sub- and intraretinal fluid. Visual acuity in the right eye decreased slightly to 0.06 and then remained unchanged for more than 1 year.

\section{Discussion}

Our 87-year-old Japanese patient with RAP developed a large tear of the RPE after vitrectomy was performed in order to release vitreomacular traction. An RPE tear is one of the complications of wet AMD, and it sometimes leads to severe visual impairment. It has been reported that an RPE tear can occur spontaneously and also after intravitreal antiVEGF drugs such as bevacizumab [9]. The reasons for the development of an RPE tear were suggested to be (1) deformation of the globe during the injection; (2) secondary vitreous traction, and (3) acute contraction of the CNV. The formation of a tear of the RPE after vitrectomy for AMD has not been reported, although it has been reported that there is an association between the development of AMD and vitreomacular traction [3]. Pars plana vitrectomy, including surgical separation of the vitreous from the retina without any manipulation of the subretinal tissue, has been used to release vitreomacular traction. This type of vitrectomy is performed to prevent further damage to the retina and RPE, which is usually observed after submacular surgery. Mojana et al. [8] reported on 5 eyes with AMD and vitreomacular traction that underwent vitrectomy; they recorded an improvement in the visual acuity by more than 1 line and a decrease of central foveal thickness in 4 of the 5 cases. Ikeda et al. [6] reported that approximately half of their cases with vitreomacular traction had an improvement in their visual acuity and reduction of the CNV in 8 of their 12 cases. There were no major complications after the less invasive surgery the patients underwent, but a retinal tear developed in the peripheral fundus.

Our case had a firm adhesion of the vitreous to the macula, and the pressure induced by aspiration of the vitreous cutter might have been transmitted to the RPE. We suggest that there were 2 major mechanisms which allowed the tractional force of the cutter to be transmitted to the RPE. First, the neurosensory retina is attached to the RPE by the interphotoreceptor matrix, which exists between the cone photoreceptors and microvilli of the apical surface of the RPE [10]. Second, RAP appeared to reach the sub-RPE space and caused detachment of the RPE [11]. Thus, the RPE and neurosensory retina were connected by neovascular tissue. The tractional force induced by the vitrectomy cutter could most likely lead to stress on the RPE at the border between the detached and undetached areas. 
Baba et al.: Retinal Pigment Epithelium Tear after Vitrectomy for Vitreomacular Traction Syndrome in an Eye with Retinal Angiomatous Proliferation

We suggest that this stress induced the small tear of the RPE which led to a larger tear after contraction of the fibrovascular tissue of the RAP.

Enzymatic vitreolysis has been reported to be effective in treating vitreomacular traction syndrome including idiopathic macular holes [12]. Ocriplasmin is a recombinant protease which shows proteolytic activity against laminin and fibronectin, the major components of the vitreoretinal interface. In the future, it will be possible to release vitreoretinal traction without a risk of undesirable mechanical retinal traction during vitrectomy in cases with vitreomacular traction and AMD.

Our case did not show a resolution of subretinal fluid after the formation of the RPE tear, and the patient's visual acuity remained poor postoperatively. Although we did not determine the precise mechanism which caused the tear of the RPE, surgeons should be aware that vitrectomy used to treat AMD in eyes with RAP and vitreomacular traction can cause an RPE tear postoperatively.

\section{References}

-1 Yannuzzi LA, Negrao S, Iida T, Carvalho C, Rodriguez-Coleman H, Slakter J, Freund KB, Sorenson J, Orlock D, Borodoker N: Retinal angiomatous proliferation in age-related macular degeneration. Retina 2001;21:416434.

-2 Krebs I, Glittenberg C, Zeiler F, Binder S: Spectral domain optical coherence tomography for higher precision in the evaluation of vitreoretinal adhesions in exudative age-related macular degeneration. Br J Ophthalmol 2011;95:1415-1418.

- 3 Nomura Y, Ueta T, Iriyama A, Inoue Y, Obata R, Tamaki Y, Yamaguchi T, Yanagi Y: Vitreomacular interface in typical exudative age-related macular degeneration and polypoidal choroidal vasculopathy. Ophthalmology 2011;118:853-859.

4 Schulze S, Hoerle S, Mennel S, Kroll P: Vitreomacular traction and exudative age-related macular degeneration. Acta Ophthalmol 2008;86:470-481.

-5 Lee SJ, Koh HJ: Effects of vitreomacular adhesion on anti-vascular endothelial growth factor treatment for exudative age-related macular degeneration. Ophthalmology 2011;118:101-110.

6 Ikeda T, Sawa H, Koizumi K, Yasuhara T, Yamasaki T: Pars plana vitrectomy for regression of choroidal neovascularization with age-related macular degeneration. Acta Ophthalmol Scand 2000;78:460-464.

-7 Schmidt JC, Mennel S, Horle S, Meyer CH: High incidence of vitreomacular traction in recurrent choroidal neovascularisation after repeated photodynamic therapy. Br J Ophthalmol 2006;90:1361-1362.

8 Mojana F, Cheng L, Bartsch DU, Silva GA, Kozak I, Nigam N, Freeman WR: The role of abnormal vitreomacular adhesion in age-related macular degeneration: spectral optical coherence tomography and surgical results. Am J Ophthalmol 2008;146:218-227.

-9 Meyer CH, Mennel S, Schmidt JC, Kroll P: Acute retinal pigment epithelial tear following intravitreal bevacizumab (Avastin) injection for occult choroidal neovascularisation secondary to age related macular degeneration. Br J Ophthalmol 2006;90:1207-1208.

$\checkmark 10$ Hollyfield JG, Varner HH, Rayborn ME, Osterfeld AM: Retinal attachment to the pigment epithelium. Linkage through an extracellular sheath surrounding cone photoreceptors. Retina 1989;9:59-68.

11 Freund KB, Ho IV, Barbazetto IA, Koizumi H, Laud K, Ferrara D, Matsumoto Y, Sorenson JA, Yannuzzi L: Type 3 neovascularization: the expanded spectrum of retinal angiomatous proliferation. Retina 2008;28:201211.

12 Stalmans P, Benz MS, Gandorfer A, Kampik A, Girach A, Pakola S, Haller JA: Enzymatic vitreolysis with ocriplasmin for vitreomacular traction and macular holes. N Engl J Med 2012;367:606-615. 


\section{Case Reports in \\ Ophthalmology}

\begin{tabular}{l|l}
\hline \multicolumn{2}{l|}{ Case Rep Ophthalmol 2013;4:165-171 } \\
\hline DOI: $10.1159 / 000355829$ & $\begin{array}{l}\text { ○ 2013 S. Karger AG, Basel } \\
\text { www.karger.com/cop }\end{array}$ \\
\hline
\end{tabular}

Baba et al.: Retinal Pigment Epithelium Tear after Vitrectomy for Vitreomacular Traction Syndrome in an Eye with Retinal Angiomatous Proliferation
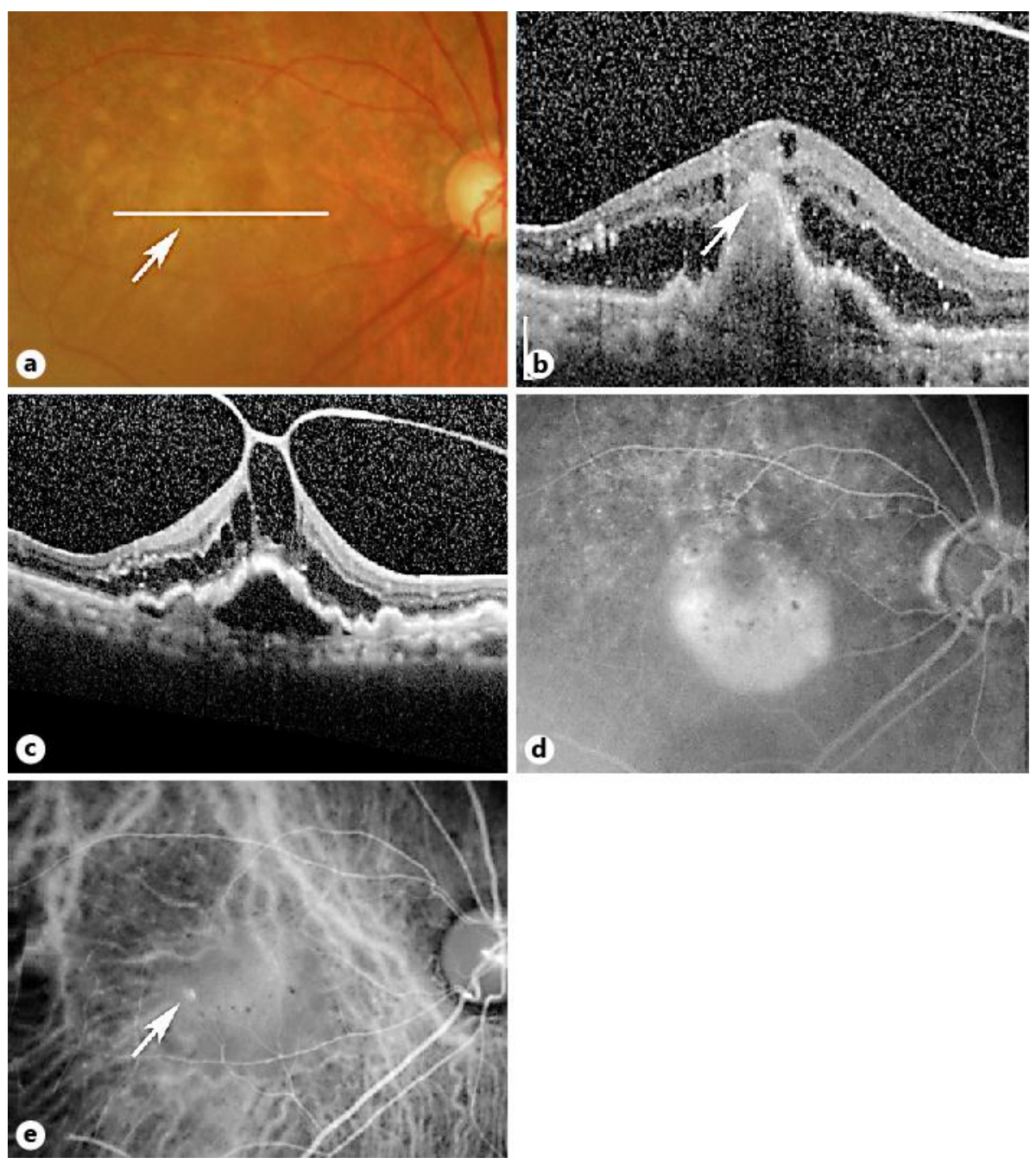

Fig. 1. Fundus photographs, OCT images, and fluorescein and indocyanine green angiograms in a patient with RAP and an RPE detachment. a An RPE detachment, including the fovea, can be seen with multiple drusen. A small intraretinal hemorrhage can be seen (arrow). The patient's visual acuity was 0.15 . b OCT image shows a hyperreflective mass above the RPE (arrow) and intra- and subretinal fluid. The location of the scan is shown by a white line in a. c OCT image through the fovea showing the RPE detachment and vitreomacular adhesion. $\mathbf{d}$ Late-phase fluorescein angiogram shows a pooling of dye at the site of the RPE detachment. e Indocyanine green angiogram shows a hyperfluorescent spot temporal to the fovea (arrow). 


\section{Case Reports in Ophthalmology}

Case Rep Ophthalmol 2013;4:165-171

DOI: $10.1159 / 000355829$

Baba et al.: Retinal Pigment Epithelium Tear after Vitrectomy for Vitreomacular Traction Syndrome in an Eye with Retinal Angiomatous Proliferation
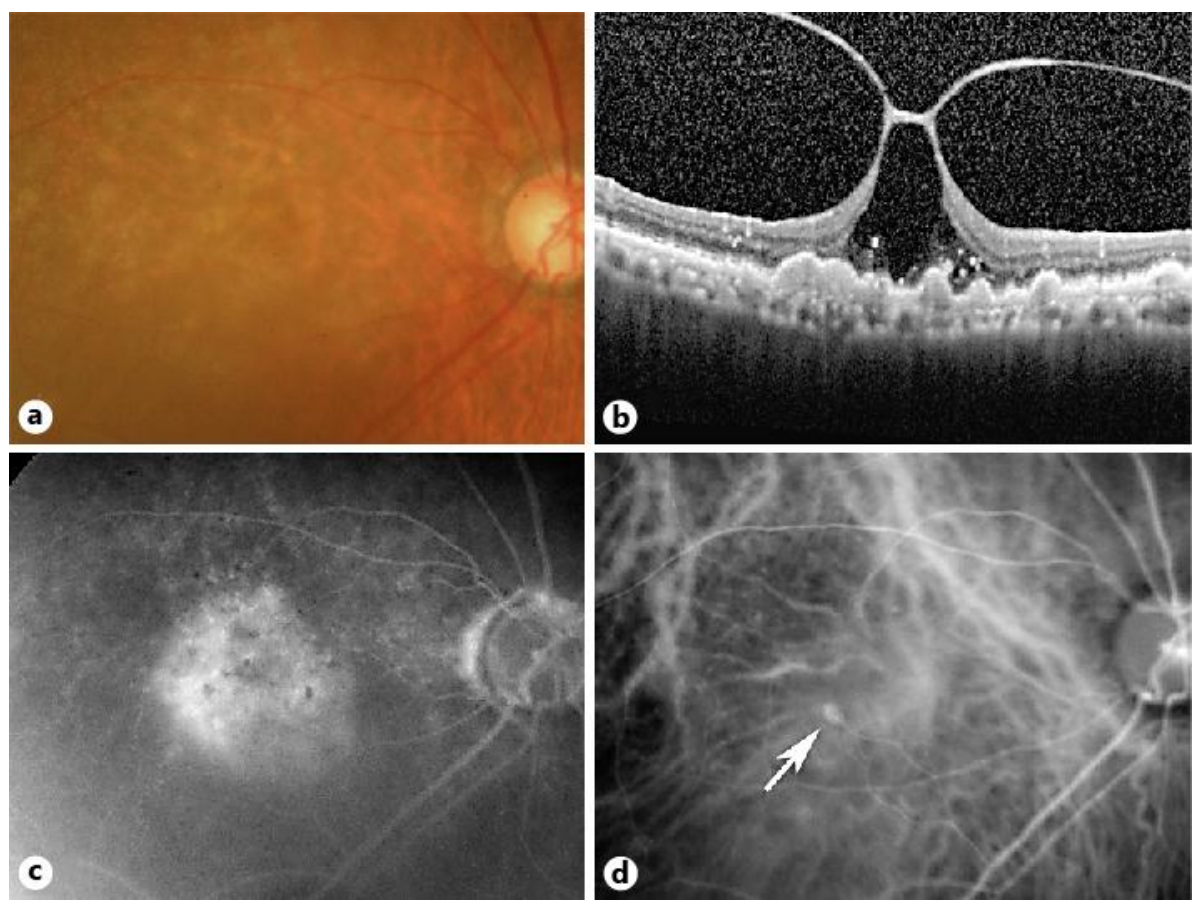

Fig. 2. Fundus photographs, OCT image, and fluorescein and indocyanine green angiograms in a patient with RAP and an RPE detachment after 3 monthly injections of ranibizumab. a Fundus image after 3 monthly intravitreal injections of ranibizumab. The RPE detachment is reduced, and the patient's visual acuity improved slightly to 0.2 . b OCT image shows a resolution of the RPE detachment. However, the vitreous adhesion to the macula is still present, causing an abnormal shape of the fovea. c Late-phase fluorescein angiogram shows a decrease in the pooling of the dye. $\mathbf{d}$ Indocyanine green angiogram shows a small hyperfluorescent spot (arrow). The leakage from a retinal choroidal anastomosis is weaker. 


\section{Case Reports in \\ Ophthalmology}

\begin{tabular}{l|l}
\hline Case Rep Ophthalmol 2013;4:165-171 \\
\hline DOI: 10.1159/000355829 & $\begin{array}{l}\text { ○ 2013 S. Karger AG, Basel } \\
\text { www.karger.com/cop }\end{array}$ \\
\hline
\end{tabular}

Baba et al.: Retinal Pigment Epithelium Tear after Vitrectomy for Vitreomacular Traction Syndrome in an Eye with Retinal Angiomatous Proliferation
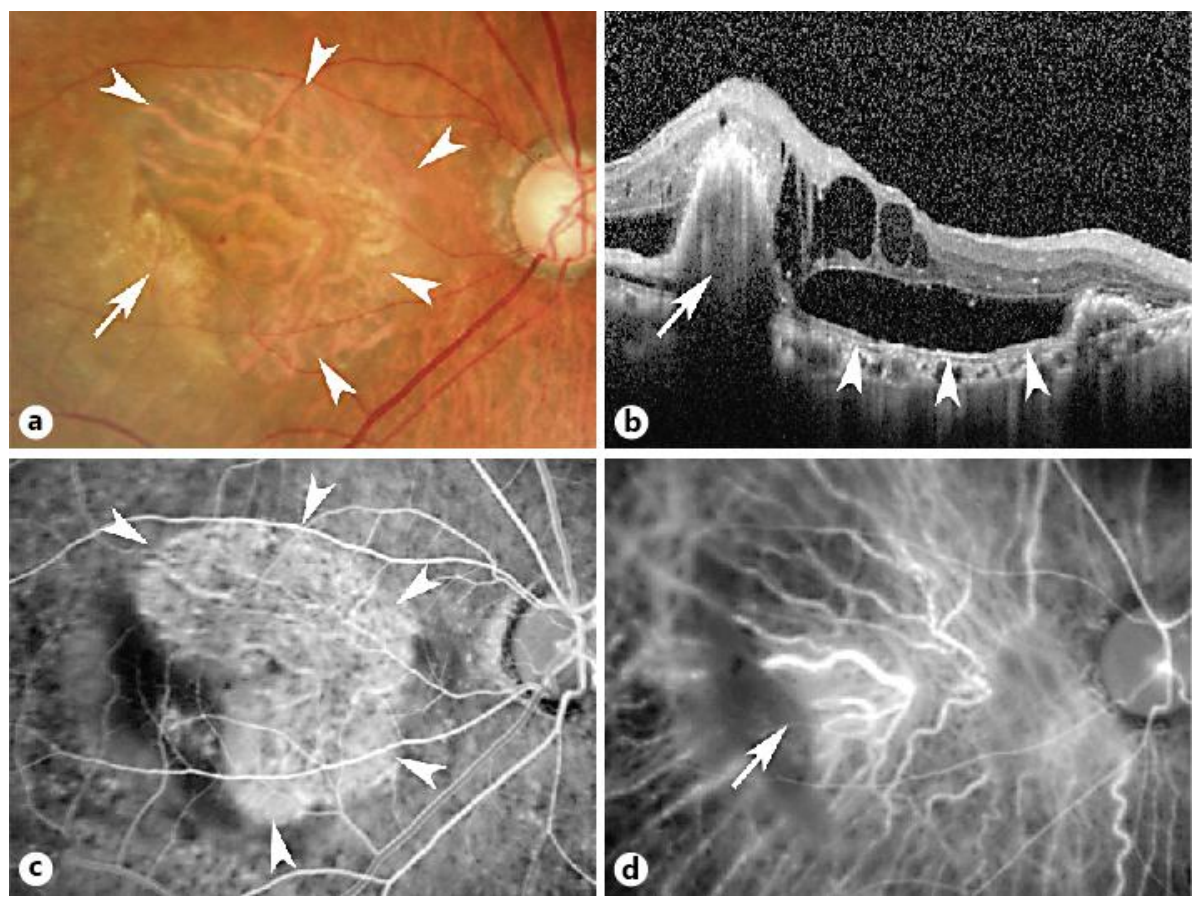

Fig. 3. Fundus photographs, OCT image, and fluorescein and indocyanine green angiograms in a patient with RAP and an RPE tear that developed 2 months after vitrectomy for vitreomacular traction. a Fundus photograph 2 months after the vitrectomy. Note the large defect of the RPE (arrowheads) and the rolled epithelium (arrow) temporal to the fovea. b OCT image shows an accumulation of intra- and subretinal fluid. The RPE is rolled (arrow), and the area not covered by the RPE can be seen (arrowheads). c Fluorescein angiogram shows an area of hyperfluorescence corresponding to the RPE tear (arrowheads). $\mathbf{d}$ Indocyanine angiography shows the neovascular lesion covered by the contracted RPE (arrow). 